\title{
Working party 3 session: foods and people
}

\section{Cost effectiveness issues in relation to the implementation of EU food based dietary guidelines}

\author{
Eric Brunner ${ }^{\star 1}$, David Cohen ${ }^{2}$, Lynn Toon ${ }^{1}$
}

1 International Centre for Health and Society, Department of Epidemiology and Public Health, University College London Medical School, 1-19 Torrington Place, London WC1E 6BT, England

${ }^{2}$ Business School, University of Glamorgan, Pontypridd, CF37 1DL, Wales

* Correspondence: Email e.brunner@ucl.ac.uk

Keywords primary prevention; public health; diet; costs and cost analysis

For policymakers considering strategy options for the prevention of cardiovascular disease (CVD) the distinction between effectiveness and cost effectiveness is critical. When cost limitations apply, an evaluation of cost effectiveness is essential if a rational decision is to be made. Policy changes and resource reallocation have opportunity costs, and therefore it is necessary to compare the cost of health gains achievable by means of different policies. Here the broad question is: How cost effective are diet change strategies compared to other measures aimed at reducing cardiovascular disease in $\mathrm{EU}$ member states?

An overview of published studies of costeffectiveness in the primary and secondary prevention of cardiovascular disease was conducted. Few comprehensive studies were available.

Estimated costs per life year gained were as follows: population-based healthy eating $£ 14-560$; smoking cessation $£ 300-790$; nurse screening and life style advice $£ 900$ (minimum); simvastatin (HMGCoA reductase inhibitor) $£ 6200-11,300$. Cost effectiveness is dependent on the underlying level of CVD risk in the target population, and the duration of the achieved alterations in behaviours and risk factors.

The limited evidence from these studies tends to support the view that health protection strategies which promote healthy eating are likely to be more cost-effective than strategies involving modern cholesterol-lowering drugs, screening and advice in primary care, and are comparable to or less expensive per year of life saved than anti-smoking strategies. Given the considerable diversity in food habits, health care and public health systems among current and prospective EU member states, careful appraisal of the policy options within each member state is desirable to ensure that health gain is maximised. EU wide food based dietary guidelines are potentially the basis of large health gains in Europe, and cost-effectiveness studies tend to support their adoption.

\section{Promoting physical activity: 2 case studies}

Johan De Rycker*

Université Libre de Bruxelles, Department of Journalism and Communication and observer to the Working Party for

Confédération des Industries Agro-Alimentaires de I'UE (C.I.A.A.)

* Correspondence: Email johan-de-rycker@tnn.raftir.be

Keywords physical activity, health care setting, educational setting

Objectives: The purpose is to present two case studies on promoting Physical Activity in a health care setting (France) and an educational setting
(Belgium), in order to highlight both the criteria for success of such activities and the obstacles that may lead to failure. 
1. Physical Activity Prescription (PEP'S, France)

In this programme, Programme d'Evaluation Physique et Sportive (PEP'S) a two-part survey is first completed (one for the general practitioner (GP) or health care professional, one for the patient). The GP (or health care professional) then receives a personalised physical activity prescription programme, for his/her patient. Initial results indicate that this programme is more effectively used by health care professionals other than GPs.

2. Physical Activity Promotion (SportDuel, Belgium)

This programme (a strategic, physical activity, nutritional knowledge board game) started with a competition among schools where adolescents were asked to develop a tool for promoting physical activity in their schools. The winning concept was then further developed by an interdisciplinary team into this board game. Involving all the relevant parties in this school setting from the start, has meant that this programme is now being used not only in schools, but also in other adolescent settings (organised family association settings, organised leisure settings).

Conclusions. The key to the success of these actions was the original insights that came from the target groups themselves combined with the subsequent development of the programmes with representatives of all relevant setting stakeholders right from the start. The obstacles that needed to be overcome were more related to the self-image of some of the stakeholders and the patience and commitment needed by all to see such a (lengthy) process through.

\section{School meals, a key element for the implementation of dietary guidelines in the school setting}

\section{Carmen Perez-Rodrigo}

Community Nutrition Unit. Department of Public Health, Luis Brinas 18-30, E48013, Bilbao, Spain

* Correspondence: Email bisaludpublica@jet.es

The school setting provides a valuable opportunity to influence health through policy measures, education and food provision.

The first School Meals Survey carried out in Bilbao in 1984 led to the development of the Community Nutrition Unit based in the Local Department of Public Health. Further community nutrition diagnosis of school children in Bilbao identified different situations in the city. Wide diffusion of the results contributed to increasing the awareness of school masters, teachers, social workers and school health people in the districts, encouraging involvement in an action plan. On the basis of the results, different strategies were designed including school meals; planning and implementation of nutrition education in schools; training for teachers and other people working in schools; collaboration with parents associations, school kitchen personnel and catering companies operating in schools, and collaboration with local mass media communicating nutrition and health aspects to the public.

All these activities have been carried out sequentially, including a School Meals Surveillance and Monitoring Program. Qualitative and quantitative dimensions of the evaluation show higher acceptance of fruit, vegetables and pulses. Results show notable changes, but also several aspects to be improved regarding the provision of food at schools. 
School age is an interesting stage to promote healthy food habits, encouraging the attitudes, skills and behaviours required. Fostering nutrition education in the school curriculum is a requirement, actively involving teachers, family and other community professionals. In this context, school meals should be considered as part of the educational process. National policies and regulations to support this process and ensure the provision of adequate resources are required.

\title{
Breastfeeding In Europe - rationale and prevalence
}

\author{
Agneta Yngve*, Michael Sjöström
}

Unit for Preventive Nutrition, Dept of Biosciences at Novum, Karolinska Institutet and Stockholm County Council, S-141 57

Huddinge, Stockholm, Sweden and Dept of Physical Education and Health, University of Örebro, Sweden

* Correspondence: Email agneta.yngve@prevnut.ki.se

Keywords

breastfeeding, infant feeding, breastfeeding prevalence, exclusive breastfeeding, infant nutrition

Objectives Both short and long-term health benefits exist from breastfeeding, including prevention of infections and chronic diseases as well as a favourable cognitive development. A number of consensus statements at international level have been produced, all of which agree on the importance of exclusive breastfeeding (breastfeeding without any food substitutes).

The recommendation, which is most often referred to, is found in the Innocenti Declaration

A European consensus statement on the importance of breastfeeding for optimal health of mother and child is still lacking, as well as an official EU recommendation regarding the optimal duration of exclusive and partial breastfeeding. In our work with the background document for the breastfeeding guidelines recommended by the Eurodiet project, it became clear early on that breastfeeding in the European region is a relevant public health issue where there is a huge need, but also potential, for improvements.

Results Great differences seem to exist in breastfeeding prevalence and duration both between and within countries. Trends point towards higher prevalence and duration, although there are some exceptions. Young mothers seem to breastfeed less than older mothers, whilst single and/or lower educated women breastfeed less than married women with higher socioeconomic status.
(1992) 1. It claims exclusive breastfeeding for 4-6 months. More recent recommendations do exist. In 1999, UNICEF published an advocacy booklet recommending exclusive breastfeeding for about six months ${ }^{2}$. At a recent WHO/UNICEF Technical Consultation on Infant Feeding (March 13 to 17, 2000), the experts expressed an informal consensus that the appropriate age for recommendation of complementary feeding is "about six months" 3 .

However, inefficient and unreliable monitoring systems prevail. Prevalence data were found not to be regularly reported to the international data bases at WHO Europe ${ }^{4}$ and WHO Geneva ${ }^{5}$. A number of difficulties appeared when trying to identify comparable data. Neither the definitions of breastfeeding nor the age of child when data were collected were consistent between countries. Furthermore, the data were not always published in peer reviewed journals. Data on exclusive breastfeeding as such were seldom collected.

A minority of European countries had promotion of breastfeeding incorporated into their national plan of action for nutrition ${ }^{6}$. Officially appointed national breastfeeding co-ordinators were found in only a few countries?.

Conclusions Differences in breastfeeding prevalence and duration between and within countries, as well as between different socio-economic groups, need to be reduced. However, to be able to effectively promote exclusive breastfeeding in Europe, a 
number of elements need to be in place at European and/or national level;

Effective and comparable surveillance systems over Europe

A strong consensus statement, formulated in Europe for Europe regarding the benefits of breastfeeding for both mother and child

A common European recommendation for breastfeeding duration and exclusiveness building on the above consensus statement

A European action plan to promote breastfeeding Revised national policies/plans of action for nutrition/breastfeeding, including a national breastfeeding co-ordinating function, with sufficient resources for action.

\section{References}

1. WHO/UNICEF. The Innocenti Declaration on the protection, promotion and support of breastfeeding, Geneva: WHO/UNICEF, 1990.

2. UNICEF. Breastfeeding: foundation for a healthy future. Copenhagen: UNICEF Geneva Region, 1999.

3. Ferriman A. WHO accused of stifling debate about infant feeding. BMJ 2000; 320: 1362.

4. WHO Regional Office for Europe. Health For All Data Base, European Region. Copenhagen: WHO, 1999.

5. WHO Headquarters Nutrition Unit. Global Data Bank on Breast-feeding. Geneva: WHO 1996.

6. WHO Europe, Nutrition Policy in WHO European member states. Copenhagen: WHO Nutrition Unit, 1995.

7. WHO Regional Office for Europe, Headquarters and United Nations Children's Fund, Comparative Analysis of Implementation of the Innocenti Declaration in WHO European Member States Geneva: WHO/UNICEF,1998. 